\title{
The effect of surface orientation on spray retention
}

\author{
Massinon M., Boukhalfa H.H., and Lebeau F. \\ Precision Agriculture Unit - Gembloux Agro-Bio Tech (ULg) \\ 2 Passage des Déportés - 5030 Gembloux (Belgium)
}

\begin{abstract}
Research in precision spraying investigates the means to reduce the amount of herbicide applied by directing droplets more accurately towards the weeds. The trend in the development of spot spraying equipment is an increase of the spatial resolution and new actuators that are able to target very small areas. However, there is a lack of methods for assigning rates of herbicides relating target to optimal droplet features. A wide range of droplet impact angles occurs during the spray application process because of droplet trajectories and the variability of leaf orientation. In this study, laboratory experiments were conducted to highlight the effect of surface orientation on droplet impact outcomes (adhesion, rebound or splashing) on two very difficult-to-wet surfaces: an artificial surface with a regular roughness pattern and an excised black-grass leaf with an anisotropic roughness pattern. Measurements were performed for different surface orientations with a high-speed camera coupled with backlighting LED. Droplets of two formulations (distilled water and distilled water + a surfactant) were produced with a moving flat-fan hydraulic nozzle to obtain a wide range of droplet sizes and velocities, which were measured by image analysis. Increasing surface angle reduces surface area available for droplet capture. Droplet impact behaviors are then modified since surface tilt induces a tangential velocity component at impact and, consequently, a reduction of the normal component. Impact modifications have also been observed due to the anisotropic roughness pattern of a black-grass leaf. The integration of droplet-surface interaction information offers a significant way to further improve the precision spraying efficiency by considering the optimal droplet size, speed and ejection angle depending on the target surface and architecture.
\end{abstract}




\section{Keywords}

Precision spraying, Spray retention, Leaf orientation, Droplet impact, High-speed imaging, Spot spraying

\section{Introduction}

Chemical weed control continues to play a pivotal role to ensure a sufficient level of yield. Agrochemical use, however, is associated with ever increasing environmental, safety and cost concerns. Targeting pesticides more accurately is then of major concern for reducing the negative impact of weed chemical control. Conventional uniform application with hydraulic nozzles was optimized over many years to achieve better spray distribution over the crop and better biological efficiency while minimizing spray drift. Studies aimed at finding the best use of this spraying equipment depending on applied volumes, pesticide dose and crop growth stage and species, most often using field trials (Butler Ellis et al. 2004). Regarding spraying equipment, the development of the so-called controlled droplet application allowed reduction of the volume of pesticides per hectare applied with emphasis on the importance of applying the correct size of droplets for a given target with uniform droplet size distributions (Matthews 2008). Besides, the notion of differential management of field areas derived from precision farming concepts has further resulted in a more precise application technique consisting of applying control measures only where weeds are located. The first advance was the introduction of variable rate technology that controls the applied amount of pesticide according to site-specific demand in the field based on spray maps generated before the treatment. This method requires large scale remote sensing techniques to build offline spray application prescription maps later used in the field to drive GPS spray controllers. Precision agriculture flow control has recently evolved to high resolution machine vision detection systems allowing real-time capabilities (Thorp and Tian 2004). The detection and identification of individual weeds requires both high resolution machine vision technology systems (Tellaeche et al. 2008; Nieuwenhuizen et al. 2010) and an actuator for pesticide, delivering to the right target (Lee et al. 1999; Giles et al. 2002; Miller et al. 2012a).

The main factor driving the development of spot spraying equipment is to maximize the number of droplets reaching their target. Droplet transport 
(Walklate 1987; Ghosh and Hunt 1998) and spray drift potential (Holterman et al. 1997; Mokeba et al. 1997; Baetens et al. 2007; Butler Ellis and Miller 2010) were described intensively and many solutions were proposed to reduce their adverse effects. However, mechanisms governing spray retention, which is the amount of sprayed product actually retained by plant leaves, are still misunderstood despite the growing interest of researchers. On their part, the losses occurring during droplet impact on leaves amount to 5 - $92 \%$ of the off-target component load of a herbicide application (Zabkiewicz 2007). Miller et al. (2012b), in a quest for a suitable nozzle to perform plant scale control for use in spot spraying applications, highlighted that the use of larger and faster droplets in the spray gave an additional component of selectivity: retention on the onion crop was reduced from $6.35 \mu 1$ for the nozzle delivering the slower and smaller droplets to $0.87 \mu 1$ for the bigger and faster ones, a 7.5:1 ratio, while retention on filter paper was only modified in a 2:1 ratio. This means that, even if droplets impact their target, treatment efficiency differences may arise from retention variability depending on plant (species, wettability, growth stage and architecture) and droplet properties (size, velocity, dynamic surface tension). This has to be taken into account for development of spot spraying equipment and advantage can even be taken from differences of wettability between species for targeting specific weeds or reducing contamination of crops. The need for further investigation on the effect of coverage, dosage and placement on the leaves was also highlighted for further micro-spray systems development (Søgaard and Lund 2007). The next gap to fill in the development of variable-rate technologies is the lack of methods for assigning rates of herbicide based on the results of a weed sensing procedure (Thorp and Tian 2004). A better knowledge of droplet-leaf interactions by precision farming specialists is therefore requested to drive the development of the next generation of pesticide application technology.

Many parameters influence droplet behavior at impact, from droplet physicochemical properties to target properties. Predominant factors affecting the spray retention are related to the target surface (Furmidge 1962; Wirth et al. 1991; Journaux et al. 2011). Plant leaves exhibit various degrees of wettability from very-easy to very-difficult-to-wet, depending on species and growth stages (Gaskin et al. 2005) owing to the coating of epicuticular wax at leaf surface (Barthlott et al. 1998). Surface wettability is often quantified using the static 
contact angle $\theta$, which is the angle measured between the solid surface and the tangent to droplet at the point where liquid, solid and air meet. It reflects the relative strength of the liquid, solid and vapor molecular interactions. High static contact angle $\left(90^{\circ}<\theta<150^{\circ}\right)$ reflects hydrophobic surfaces. Static contact angle above $150^{\circ}$ determines superhydrophobic surfaces. The superhydrophobic behavior of some leaves arises from the presence of a microstructure of the epicuticular wax. The superhydrophobicity is therefore a physical property of hydrophobic materials. In other words, the hydrophobicity provided to some leaves by waxes is enhanced by hairs that dramatically increase the small scale roughness of the leaf surface. In consequence, a droplet exhibits a very large static contact angle on such surfaces and is not able to stay on it at impact. It is well established that superhydrophobic species, such as blackgrass, are the most challenging target for efficient pesticide application. The present paper focuses therefore on such surfaces.

Two models describe the wetting of such surfaces based on Young's equation and liquid surface tension (Callies and Quere 2005; Taylor 2011) at microscopic scale. In the Wenzel non-composite regime (Wenzel 1936), often referred to as homogeneous wetting regime, the liquid wets and fills the surface cavities completely thanks to a sufficiently low liquid surface tension. The Cassie-Baxter composite regime (Cassie and Baxter 1944) considers an interface composed of both solid and surrounding air trapped under the drop ( $\mathrm{Zu}$ et al. 2010). The liquid is only in contact with the upper part of the relief because its surface tension is too high. Height and distance between pillars or pikes that make up the roughness of the surface are critical parameters to keep the drop in the Cassie-Baxter regime. A transition between the Cassie-Baxter to the Wenzel regime is possible and may be caused by droplet surface fluctuations when the droplet is resting on the surface at impact.

On superhydrophobic leaves, the outcome of droplet impact is a complex function of surface roughness, surface orientation, droplet size and velocity and liquid physico-chemical properties. Many studies focused on optimal droplet size required maximization of retention by plants under field conditions (Knoche 1994; Butler Ellis et al. 2004). However, systematic laboratory tests are needed to gain more precise information at droplet scale. Impact outcomes of a single droplet on horizontal and dry superhydrophobic surfaces are known to be a 
function of droplet Weber number and surface roughness (Rein 1993; Yarin 2006). The Weber number represents the ratio of droplet kinetic energy to surface energy (Eq. 1).

$$
W e=\frac{\rho v^{2} d}{\sigma}
$$

where $\mathrm{d}$ is the droplet diameter $[\mathrm{m}], v$ is the droplet velocity $\left[\mathrm{m} \mathrm{s}^{-1}\right], \rho$ is the liquid density $\left[\mathrm{kg} \mathrm{m}^{-3}\right]$ and $\sigma$ is the liquid surface tension $\left[\mathrm{N} \mathrm{m}^{-1}\right]$. The Wenzel's roughness factor, defined as the ratio of actual and projected planar or geometrical (measured in the plane of the interface) surface areas (Rioboo et al. 2008), indicates the surface roughness level. This ratio tends to one for a flat and smooth surface. For small Wenzel roughness and for low Weber number (Fig. 1), a drop is deposited in the Wenzel regime. Higher Weber numbers lead to drop fragmentation, part of which sticks at the impact point. Depending on impact energy, a droplet can either bounce which is called partial rebound, or shatters into several satellite drops, which is called partial splashing. For intermediate Wenzel roughness, slow drops adhere in a Cassie-Baxter regime. For higher Weber number, the droplet bounces completely, which is only possible on superhydrophobic surfaces (Quéré 2005). For even higher Weber number, impact pressure is so large that the liquid can penetrate surface cavities, which modifies the wetting regime from Cassie-Baxter to Wenzel. Transition from Cassie-Baxter to Wenzel wetting regime may also occur by reducing liquid surface tension. As a consequence, sticking, partial rebound or partial splashing may occur. Finally, for high Wenzel roughness, drops may either adhere in a Cassie-Baxter regime, rebound or splash completely depending on Weber number.

Fig. 1 Possible droplet impact outcomes on a superhydrophobic surface depending on surface roughness and droplet impact velocity

The structure of surface micro-topography further modifies liquid macroscopic wetting. On unidirectional grooved surfaces, contact angles measured from the direction parallel to the grooves are larger than those measured from the perpendicular direction (Zhao et al. 2007). Surface micro-pattern also influences the macroscopic flows as directional splashing can occur (Tsai et al. 2011). 
Treating superhydrophobic leaves is a challenge accentuated by the angle of droplet impact due both to plant architecture and droplet trajectories. Jensen (2012) showed that angling a nozzle at $60^{\circ}$ forward relative to the direction of travel increased herbicide efficacy on annual grasses, such as blackgrass, using flat-fan nozzles in field experiments. Obviously, surface angle reduces projected area intercepting droplet spray. Up to now, the effect of surface angle on droplet impact outcome has been weakly documented. Most studies (Stow and Hadfield 1981; Mundo et al. 1995; Šikalo et al. 2005; Bird et al. 2009) advocated the use of velocity normal component in computing dimensionless numbers to forecast impact outcome thresholds (Lake and Marchant 1983). The optimal droplet features depending on those of the target are required to guide the further development of precision spraying technology. This paper shows how to consider the droplet angle at impact to find this optimum using a laboratory method devised to rank adjuvants according to their effect on retention in controlled conditions (Massinon and Lebeau 2012b). More particularly, this study focuses on how leaf orientation may affect retention by plants. First, an artificial superhydrophobic surface was used to investigate adjuvant effect on retention as a function of surface angle. The artificial surface was used as a reference for systematic tests since variability owing to leaf position and age could hide differences between impact behaviors (Reichard et al. 1998). Afterwards blackgrass, a common weed in cereal crops, was investigated to see whether mechanisms highlighted on an artificial surface can be extended to natural surfaces.

\section{Materials and methods}

\section{Dynamic spray application bench}

\section{Spraying system}

The setup (Fig. 2) was composed of a dynamic spray application test bench contained in a room where temperature and relative humidity were controlled. The dynamic bench consisted of a single extended range flat-fan nozzle (XR Teejet 11003 VK, Spraying Systems Co, Wheaton, IL, USA) operating at $200 \mathrm{kPa}$. The nozzle was mounted $500 \mathrm{~mm}$ vertically above the target surface on a linear guide rail actuated by a servomotor at a forward speed of $2 \mathrm{~m} \mathrm{~s}^{-1}$. A single pass was 
performed for each spraying so that a volume of $1601 \mathrm{ha}^{-1}$ was delivered during tests.

Fig. 2 Dynamic spray application bench: (1) high-speed camera (2) LED lighting (3) target surface on linear stage (4) computer (5) pressurized tank (6) solenoid valve (7) nozzle (8) pressure gauge (9) servomotor (10) programmable controller and (11) linear stage

\section{Backlit imaging system}

Drop impacts were recorded with a high-speed camera (Y4, Integrated Design Tools, Tallahassee, FL, USA) at a frequency of 20000 frames per second with a 9 $\mu \mathrm{s}$ exposure time to provide experimental information on number, size and velocity of drops before impact, as well as drop impact behavior. An optical system (12X zoom system, Navitar, Rochester, NY, USA) provided 1016x185 pixels digital images with $11.3 \mu$ m.pixel ${ }^{-1}$ spatial resolution, calibrated using the United States Air Force resolution test chart (MIL-STD-150A, section 5.1.1.7) and a camera depth of field at about $2 \mathrm{~mm}$. Consequently, number density and spatial statistics were given from a probe volume of $11.48 \times 2.09 \times 2 \mathrm{~mm}$ (image size $x$ depth of field). The setup involved a pulsed LED lighting array (19-LED Constellation, Integrated Design Tools, Tallahassee, FL, USA), which was synchronized with the image acquisition in a backlit arrangement. Distance between LED array and camera was $500 \mathrm{~mm}$. No heating problems arose in the probe volume. Light intensity on images was expressed in 8 bits grey levels with pixel value ranging from 0 for black to 255 for white. The whole optical system was tilted with respect to the target from 0 to $60^{\circ}$, so that image width was kept parallel to the surface independently of the angle of surface inclination.

\section{Image analysis}

The image processing technique used for measuring droplet size and velocity was non-intrusive. Owing to spray density, some droplets were located outside the probe volume. Such defocused drops were rejected since they represented a 
source of error. Similarly, droplets truncated by image edge or too small particles were not integrated in the procedure.

Image processing began with an automated identification of relevant images based on droplet movement to reduce the number of images to be treated. Since robust automated impact type identification was not available yet, pre-selected images were viewed by a human operator who recorded subsequent impact behavior on the basis of Fig. 1. An image pair was selected for each droplet where the first image contained an incoming droplet at its top, with its center at approximately $1.3 \mathrm{~mm}$ from the target (depending on droplet size), and the second contained the droplet immediately before impact, the bottom of the droplet being at a distance depending on the droplet velocity and the camera acquisition rate. The algorithm loaded the first image and performed a background correction to enhance image quality and reduce background illumination inhomogeneities (based on 50 images without any droplet). Then the image was binarized and particles were detected and identified. Only in-focus droplets were selected based on gradient detection at the edges of droplets (Lecuona et al. 2000). The droplet diameter was computed using the Eq. 2:

$$
d=\sqrt{\frac{4 A}{\pi}}
$$

where A was the surface area calculated by summing all pixels belonging to the droplet. If several droplets appeared in the image due to a splashing event (10-20 droplets) or multiple incoming droplets (up to 5 droplets), the operator was prompted to select the droplet of interest on the two images by clicking on it with the computer mouse. The algorithm found the nearest droplet from the coordinates of the click. Droplet center co-ordinates $(\mathrm{X}, \mathrm{Y})$ are saved for subsequent determination of droplet velocity. An identical operation was performed with the second image. The droplet velocity was then computed using Eq 3:

$$
\vec{v}_{i j}=\frac{\left(X_{j}-X_{i}, Y_{j}-Y_{i}\right)}{\Delta t}
$$

where subscripts $i$ and $j$ were respectively related to the drop on the first and second image, $\mathrm{X}$ and $\mathrm{Y}$ were the center co-ordinates and $\Delta t$ is the time interval between both selected images. The time between the two images of a pair was maximized to reduce the uncertainties on the droplet velocity determination, with the assumption that the computed velocity is the droplet velocity at impact on the 
target. The norm of the velocity vector computed in Eq. 3 was further used to build the results.

\section{Results}

\section{Experimental conditions}

Tap water constitutes the main carrier of agrochemicals. Since water hardness modifies surface tension, pure distilled water (static surface tension of $72.10^{-3}$ $\mathrm{N} \mathrm{m}^{-1}$ ) was firstly sprayed using the application bench. Agrochemicals mixture wettability is often improved by adding surfactants. In our experiments, mixture static surface tension was reduced to $21.10^{-3} \mathrm{~N} \mathrm{~m}^{-1}$ (CAM200, KSV Instruments, Helsinki, Finland, sessile drop method, 5 replicates) thanks to a trisiloxane tankmix surfactant (Break-Thru® S240, Evonik Industries AG, Germany) added to distilled water at the recommended use concentration $(0.1 \% \mathrm{v} / \mathrm{v})$. These superspreaders (Venzmer 2011) have a very low dynamic surface tension (DST), which inhibits droplet rebound (Massinon and Lebeau 2012b) and may increase retention. A surfactant molecule contains a hydrophilic head and a hydrophobic tail. Surfactant molecules will diffuse in water and adsorb at interfaces between air and liquid and align themselves with the tails to air and heads to the water, which reduce the surface tension. During impact on the target, the droplet is deformed. The interface is stretched and gaps in the surfactant alignment appear at the interface. Surfactant molecules will spontaneously fill the gaps to restore the equilibrium. Surfactants are characterized by their adsorption rate, or ability to fill the interface gaps more or less rapidly. Super-spreader surfactants exhibit a very fast adsorption rate. This kinetic of adsorption can be described by the DST, which is the variation over time of the surface tension. If the adsorption time is smaller than the droplet impact time, which is about $2 \mathrm{~ms}$, the droplet impact behavior can be greatly altered (Massinon and Lebeau 2013) since surfactants are able to maintain a very low surface tension.

An artificial superhydrophobic surface (completely PTFE coated microscope blade, part number X2XES2013BMNZ, Thermo Fisher Scientific Inc., Waltham, MA, USA) was first sprayed at different orientations by a rotation of the camerasurface-lighting system of $0^{\circ}, 30^{\circ}$ and $60^{\circ}$ around the axis $X$ (Fig. 2). The static contact angle of a distilled water droplet is $169^{\circ}$ on the surface (CAM200, KSV Instruments, Helsinki, Finland), which is representative of very difficult-to-wet 
leaves (Massinon and Lebeau 2012a). Ten sprayings were performed for each orientation and a new artificial surface was used for each spraying. Afterward, spray behavior was studied on excised leaf from indoor-grown blackgrass [Alopecurus myosuroides HUDS. (ALOMY), BBCH 13], which is a verydifficult-to-wet species (distilled water static contact angles around $155^{\circ}$ ) depending on position on leaf and surface groove direction. Five sprayings were performed on the adaxial leaf surface, which was positioned along the axis $\mathrm{Y}$ (Fig. 2).

\section{Retention by reference artificial superhydrophobic surface}

Spray impact characteristics, such as observed number of drops, volume median diameter $\left(\mathrm{D}_{\mathrm{V} 50}\right)$ and total droplets volume as well as impact characteristics are given in Table 1. Features in Table 1 were merged from ten sprayings to reduce the inherent variability existing between spraying trials, which is related to the random droplet formation process. It is recognized that about 10000 droplets are required to obtain a stabilized particle size distribution. The values of $\mathrm{D}_{\mathrm{V} 10}, \mathrm{D}_{\mathrm{V} 50}$, $\mathrm{D}_{\mathrm{V} 90}$ and number of droplets are presented in Table 1 to get an idea of the spray characteristics during the trials with the specific nozzle and formulation, but do not present values built on a sufficiently large droplets sample.

As expected for both liquids, the number of droplets landing on the surface diminishes by increasing surface angle. The observed volume reaching the surface decreases accordingly. A reducing of droplet interception by the target proportional to the cosine of the surface angle was expected. This is not observed and discrepancies between observed and projected values grow with the surface angle. For both formulations, increasing surface angle reduces adhesion and increases rebound occurrence. On an already tilted target surface, further increase of surface angle does not seem to have any effect on adhesion proportion. Partial rebound (Wenzel wetting regime) is only and scarcely observed for distilled water on a horizontal surface. By increasing surface angle, the normal velocity component is reduced. There is, therefore, not enough impact pressure to overcome the energy transition threshold between Cassie-Baxter and Wenzel wetting regimes. The super-spreader solution is able to expel the air trapped in surface roughness and spreads on the surface in Wenzel wetting state thanks to its lower DST. Rebound is therefore widely reduced using the super-spreader. Some 
drops containing surfactant molecules can however still bounce off a tilted surface. This effect increases with surface angle. Both fragmentation outcomes represent together nearly half of the volume sprayed on a horizontal surface for distilled water. Fragmentation decreases as surface angle increases for water while it increases for surfactant solution because of its lower surface tension. Partial splashing is beneficial for retention since a significant proportion of the drop may stick on the surface. For water, this proportion decreases with increasing surface angle, as partial rebound. With a surfactant solution, a few drops splash totally again because of their weak DST.

An impact phase diagram of the response to an application method of a target surface is presented in Fig. 3, depending on droplet size and velocity immediately before impact on the target, taking into account real evaporation and drag force during transport. Depending on the drop impact energy, impact outcomes succeed as described in Fig. 1. The impact phase diagram (Fig. 3) is divided into eleven energy classes. Class boundaries correspond to a constant Weber number. The first limit was set to a Weber number of 0.02 . The first energy class contains drops with a Weber number below 0.02. Successive boundaries correspond to a three times increase of drop Weber number. Boundary progression was chosen to collect enough drops to compute a representative probability of occurrence for each impact outcome within classes. Weber numbers are computed with static surface tension of distilled water and velocity modulus. Since surface tension is the main drop factor involved in impact outcome, the energy scale can be used for formulation comparison.

Fig. 4 presents volume distribution maps for six trials performed for two liquids at various surface orientations. Proportions of impact outcomes relative to the total volume are computed within eleven impact energy classes. For distilled water (Fig. 4a,b,c), adhesion proportion decreases monotonically to reach zero for the sixth energy class (We<4.86) on the horizontal surface (Fig. 4a) and energy class $5(\mathrm{We}<1.62)$ at $30^{\circ}$ and $60^{\circ}$ (Fig. $4 \mathrm{~b}$ and $4 \mathrm{c}$ respectively). Adhesion is gradually substituted by rebound below these thresholds. For instance, adhesion has 55\% probability of occurrence in the third energy class on the horizontal surface (Fig. 4a). Finally, for higher Weber numbers, rebound is substituted by splashing. The rebound/splashing boundary is sharper than the adhesion/rebound transition. A splashing threshold is located between the eighth and ninth energy class for $0^{\circ}$ 
(Fig. 4a) and $30^{\circ}$ (Fig. 4b) but between ninth and tenth class at $60^{\circ}$ (Fig. 4c). For surfactant solution (Fig. 4d,e,f), the situation is quite different. As already seen in Fig. 3, total rebound is dramatically reduced in favor of adhesion. However, increasing surface angle induces a slight rise in rebound occurrence. As for distilled water, the fragmentation threshold is reduced with angle increase (eighth class at $60^{\circ}$, Fig. 4f). Due to reduction of observed droplets with increasing surface angle (Table 1), some classes are empty in the measurements (Fig. 4f).

Fig. 3 Relation between drop velocity and diameter (Teejet ${ }^{\circledR}$ XR11003VK flat fan nozzle, $200 \mathrm{kPa}$, distilled water) and impact outcomes (adhesion $\Delta$, total rebound •, partial splashing $x$ and total splashing +) ranked in eleven energy classes for the artificial super-hydrophobic surface tilted of $30^{\circ}$ from horizontal

Fig 4 Impact outcome probability as a function of energy classes on artificial super-hydrophobic surface (green: adhesion, red: total rebound, orange: partial rebound, dark blue: total splashing and sky blue: partial splashing), + volume proportion of each energy class relative to total volume observed before impact: a b c distilled water, d e f Break-Thru $® S 240$ at $0.1 \% \mathrm{v} / \mathrm{v}$ in distilled water, a d horizontal surface, $\mathbf{b}$ e $30^{\circ}$ surface angle and $\mathbf{c} \mathbf{f} 60^{\circ}$ surface angle

\section{Retention by blackgrass leaf}

The number of droplet impacts was quite low during these experiments for practical reasons. Interpretations are then difficult but the trends can however be outlined. The probability of adhesion at first impact is in the low tens whatever leaf angle for distilled water (Table 2). A slight growth of rebound proportion is observed with increasing leaf angle. Fragmentation proportion decreases accordingly, essentially in the Cassie-Baxter wetting regime. For surfactant, adhesion proportion is in the low twenties, with a slight decrease with increasing leaf angle. Rebound is non-existent on horizontal blackgrass leaf, measurements that may be related to low number of droplets in the relevant energy class for this trial. About $60 \%$ of spray volume splashes. On a horizontal surface, a high proportion of partial splashing is corroborated by the absence of rebound. By increasing leaf inclination, total splashing (Cassie-Baxter wetting regime) takes 
over partial splashing (Wenzel wetting regime) because of the lower normal velocity component, which increases splashing threshold energy.

The epidermis outermost layer of a blackgrass leaf is comprised of hairs and a structure of unidirectional grooves. This specific leaf roughness pattern clearly affects drop impact behavior compared with an artificial surface with a regular pyramidal roughness pattern. First, a slight occurrence of partial rebound is only observed on a leaf inclined at $30^{\circ}$ for both liquids, which seems to correspond to the mean hair inclination on a blackgrass leaf. Secondly, fragmentation outcomes are directional and symmetrical since secondary droplets are ejected preferentially in both ways along surface grooves. All this highlights the variability at the leaf scale.

\section{Surface comparison}

From the comparison of results for both surfaces (Table 1 and 2), increasing surface angle leads to increased rebound proportion for distilled water. The splashing energy threshold is lower on a blackgrass leaf, which increases splashing and decreases rebound volume proportions accordingly. As expected, surfactant increases adhesion at first impact compared to distilled water. The effect is more pronounced on an artificial surface than on the blackgrass leaf surface. Rebound is almost annihilated on both surfaces by decreasing DST. Rebound is replaced by adhesion on a synthetic surface and by fragmentation on the leaf surface, which was not expected. Nevertheless rebound proportion increases with increasing surface angle on both surfaces. Splashing energy threshold is also reduced owing to the directional roughness patterns of the leaf surface. The amount of liquid left on the surface after a splashing in the Wenzel regime is not negligible in the overall retention by the plant as this impact outcome occurs always for larger droplets, which represents a huge part of the total volume despite their lower occurrence. It could be interesting to relate the impact outcomes with the overall retention in further studies.

\section{Conclusions}

An experimental technique for investigating droplet behavior at impact was proposed to guide the improvements of spray application technologies needed in the context of precision farming. Concerning spot spraying, the discontinuous 
nature of application drives the development of small nozzles able to target a single plant. For such a small area, there is a temptation to use bigger droplets directed straight downwards to be less prone to drift and offer a better control of their trajectory toward the target, what may be highly detrimental to retention. A complete understanding of the behavior at impact of these droplets as a function of the plant surface and architecture is therefore highly necessary to choose the optimal compromise between droplet size, speed and ejection angle.

In this paper, the technique has been used to investigate how the spray application features may influence spray retention efficiency depending on target properties. The broad droplet size and velocity distribution of a hydraulic nozzle was used to explore a wide range of impact energy classes. The emphasis was set on the effect of droplet impact angles since a wide range of orientation can be encountered during spray application because of droplet trajectory and leaf angle variabilities. At first glance, increasing leaf angle reduces the surface area available to droplet capture by plants, which reduces retention. Droplet impact behavior is modified since surface tilt induces a tangential velocity component and, consequently, a reduction of the normal component. Reduction of the normal velocity component mitigates transition from the Cassie-Baxter to the Wenzel wetting regime during impact on a regular roughness pattern surface. A blackgrass leaf that presents an anisotropic roughness pattern favors fragmentation compared to the artificial surface where the rebound proportion is higher. Direct adhesion represents a low spray volume proportion whatever the surface tested except when using the superspreader on an artificial surface. In general, direct adhesion is almost constant with increasing surface angle. For distilled water, fragmentation proportion decreases with increasing surface angle on both surfaces. Fragmentation is essentially comprised of total splashing.

Treating grass weeds with a predominant vertical leaf orientation such as blackgrass appears to be very difficult using sprays directed more or less vertically downwards. If bigger droplets are preferred to gain trajectory controllability, a super-spreader is highly recommended to reduce losses on these superhydrophobic targets. An angled spray for maximizing the impact occurrence on the leaf should be used to further improve retention. 


\section{Acknowledgements}

This research was funded by Service Public Wallonie DG06 (Belgium) in the framework of the EUREKA (http://www.eurekanetwork.org/) project 4984

VEGEPHY.

\section{References}

Baetens, K., Nuyttens, D., Verboven, P., De Schampheleire, M., Nicolaï, B., \& Ramon, H. (2007). Predicting drift from field spraying by means of a 3D computational fluid dynamics model. Computers and Electronics in Agriculture, 56(2), 161-173.

Barthlott, W., Neinhuis, C., Cutler, D., Ditsch, F., Meusel, I., Theisen, I., \& Wilhelmi, H. (1998). Classification and terminology of plant epicuticular waxes. Botanical Journal of the Linnean Society, 126(3), 237-260.

Bird, J. C., Scott, S. H., Tsai, S. S. H., \& Stone, H. A. (2009). Inclined to splash: triggering and inhibiting a splash with tangential velocity. New Journal of Physics, 11, 063017.

Butler Ellis, M. C., \& Miller, P. C. H. (2010). The Silsoe Spray Drift Model: A model of spray drift for the assessment of non-target exposures to pesticides. Biosystems Engineering, 107(3), 169-177.

Butler Ellis, M. C., Webb, D. A., \& Western, N. M. (2004). The effect of different spray liquids on the foliar retention of agricultural sprays by wheat plants in a canopy. Pest Management Science, 60(8), 786-794.

Callies, M., \& Quere, D. (2005). On water repellency. Soft Matter, 1(1), 55-61.

Cassie, A. B. D., \& Baxter, S. (1944). Wettability of porous surfaces. Transactions of the Faraday Society, 40, 546-551.

Furmidge, C. G. L. (1962). Physico-chemical studies on agricultural sprays. IV.The retention of spray liquids on leaf surfaces. Journal of the Science of Food and Agriculture, 13(2), 127-140.

Gaskin, R. E., Steele, K. D., \& Forster, W. A. (2005). Characterising plant surfaces for spray adhesion and retention. New Zealand Plant Protection, $58,179-183$.

Ghosh, S., \& Hunt, J. C. R. (1998). Spray jets in a cross-flow. Journal of Fluid Mechanics, 365, 109-136.

Giles, D. K., Slaughter, D. C., \& Upadhyaya, S. K. (2002). Biological target sensing and sprayer control. Aspect of Applied Biology, 66, 129-138.

Holterman, H. J., van de Zande, J. C., Porskamp, H. A. J., \& Huijsmans, J. F. M. (1997). Modelling spray drift from boom sprayers. Computers and Electronics in Agriculture, 19(1), 1-22.

Jensen, P. K. (2012). Increasing efficacy of graminicides with a forward angled spray. Crop Protection, 32(0), 17-23.

Journaux, L., Simon, J. C., Destain, M. F., Cointault, F., Miteran, J., \& Piron, A. (2011). Plant leaf roughness analysis by texture classification with generalized Fourier descriptors in a dimensionality reduction context. Precision Agriculture, 12(3), 345-360.

Knoche, M. (1994). Effect of droplet size and carrier volume on performance of foliage-applied herbicides. Crop Protection, 13(3), 163-178. 
Lake, J. R., \& Marchant, J. A. (1983). The use of dimensional analysis in a study of drop retention on barley. Pesticide Science, 14(6), 638-644.

Lecuona, A., Sosa, P. A., Rodríguez, P. A., \& Zequeira, R. I. (2000). Volumetric characterization of dispersed two-phase flows by digital image analysis. Measurement Science and Technology, 11(8), 1152-1161.

Lee, W. S., Slaughter, D. C., \& Giles, D. K. (1999). Robotic Weed Control System for Tomatoes. Precision Agriculture, 1(1), 95-113.

Massinon, M., \& Lebeau, F. (2012a). Comparison of spray retention on synthetic superhydrophobic surface with retention on outdoor grown wheat leaves. Aspect of Applied Biology, 114, 261-268.

Massinon, M., \& Lebeau, F. (2012b). Experimental method for the assessment of agricultural spray retention based on high-speed imaging of drop impact on a synthetic superhydrophobic surface. Biosystems Engineering, 112(1), 5664.

Massinon, M., \& Lebeau, F. (2013). Review of physicochemical processes involved in agrochemical spray retention. Biotechnology, Agronomy, Society and Environment, 17(3), 494-504.

Matthews, G. (2008). Pesticide Application Methods (3ed.).

Miller, P., Tillett, N., Hague, T., \& Lane, A. (2012a). The development and field evaluation of a system for the spot treatment of volunteer potatoes in vegetable crops. Aspect of Applied Biology, 112, 113-120.

Miller, P., Tillett, N., Swan, T., Tuck, C., \& Lane, A. (2012b). The development and evaluation of nozzle systems for use in targeted spot spraying applications. Aspect of Applied Biology, 114, 159-166.

Mokeba, M. L., Salt, D. W., Lee, B. E., \& Ford, M. G. (1997). Simulating the dynamics of spray droplets in the atmosphere using ballistic and randomwalk models combined. Journal of Wind Engineering and Industrial Aerodynamics, 67-68(0), 923-933.

Mundo, C., Sommerfeld, M., \& Tropea, C. (1995). Droplet-wall collisions: Experimental studies of the deformation and breakup process. International Journal of Multiphase Flow, 21(2), 151-173.

Nieuwenhuizen, A. T., Hofstee, J. W., \& Henten, E. J. (2010). Adaptive detection of volunteer potato plants in sugar beet fields. Precision Agriculture, 11(5), 433-447.

Quéré, D. (2005). Non-sticking drops. Reports on Progress in Physics, 68(11), 2495-2532.

Reichard, D. L., Cooper, J. A., Bukovac, M. J., \& Fox, R. D. (1998). Using a videographic system to assess spray droplet impaction and reflection from leaf and artificial surfaces. Pesticide Science, 53(4), 291-299.

Rein, M. (1993). Phenomena of liquid drop impact on solid and liquid surfaces. Fluid Dynamics Research, 12(2), 61-93.

Rioboo, R., Voué, M., Vaillant, A., \& De Coninck, J. (2008). Drop Impact on Porous Superhydrophobic Polymer Surfaces. Langmuir, 24(24), 1407414077.

Šikalo, Š., Tropea, C., \& Ganić, E. N. (2005). Impact of droplets onto inclined surfaces. Journal of Colloid and Interface Science, 286(2), 661-669.

Søgaard, H. T., \& Lund, I. (2007). Application Accuracy of a Machine Visioncontrolled Robotic Micro-dosing System. Biosystems Engineering, 96(3), 315-322.

Stow, C. D., \& Hadfield, M. G. (1981). An Experimental Investigation of Fluid Flow Resulting from the Impact of a Water Drop with an Unyielding Dry 
Surface. Proceedings of the Royal Society of London. Series A, Mathematical and Physical Sciences, 373, 419-441.

Taylor, P. (2011). The wetting of leaf surfaces. Current Opinion in Colloid \&amp; Interface Science, 16(4), 326-334.

Tellaeche, A., BurgosArtizzu, X. P., Pajares, G., Ribeiro, A., \& FernándezQuintanilla, C. (2008). A new vision-based approach to differential spraying in precision agriculture. Computers and Electronics in Agriculture, 60(2), 144-155.

Thorp, K. R., \& Tian, L. F. (2004). A Review on Remote Sensing of Weeds in Agriculture. Precision Agriculture, 5(5), 477-508.

Tsai, P., Hendrix, M. H. W., Dijkstra, R. R. M., Shui, L., \& Lohse, D. (2011). Microscopic structure influencing macroscopic splash at high Weber number. Soft Matter, 7(24), 11325-11333.

Venzmer, J. (2011). Superspreading - 20 years of physicochemical research. Current Opinion in Colloid \& Interface Science, 16(4), 335-343.

Walklate, P. J. (1987). A random-walk model for dispersion of heavy particles in turbulent air flow. Boundary-Layer Meteorology, 39(1-2), 175-190.

Wenzel, R. (1936). Resistance of solid surface to wetting by water. Industrial \& Engineering Chemistry, 28(8), 988-994.

Wirth, W., Storp, S., \& Jacobsen, W. (1991). Mechanisms controlling leaf retention of agricultural spray solutions. Pesticide Science, 33(4), 411-420.

Yarin, A. L. (2006). Drop impact dynamics: Splashing, Spreading, Receding, Bouncing.... Annu. Rev. Fluid Mech., 38, 159-192.

Zabkiewicz, J. A. (2007). Spray formulation efficacy - holistic and futuristic perspectives. Crop Protection, 26(3), 312-319.

Zhao, Y., Lu, Q., Li, M., \& Li, X. (2007). Anisotropic Wetting Characteristics on Submicrometer-Scale Periodic Grooved Surface. Langmuir, 23(11), 62126217.

Zu, Y. Q., Yan, Y. Y., Li, J. Q., \& Han, Z. W. (2010). Wetting Behaviours of a Single Droplet on Biomimetic Micro Structured Surfaces. Journal of Bionic Engineering, 7(2), 191-198. 
Figure 1

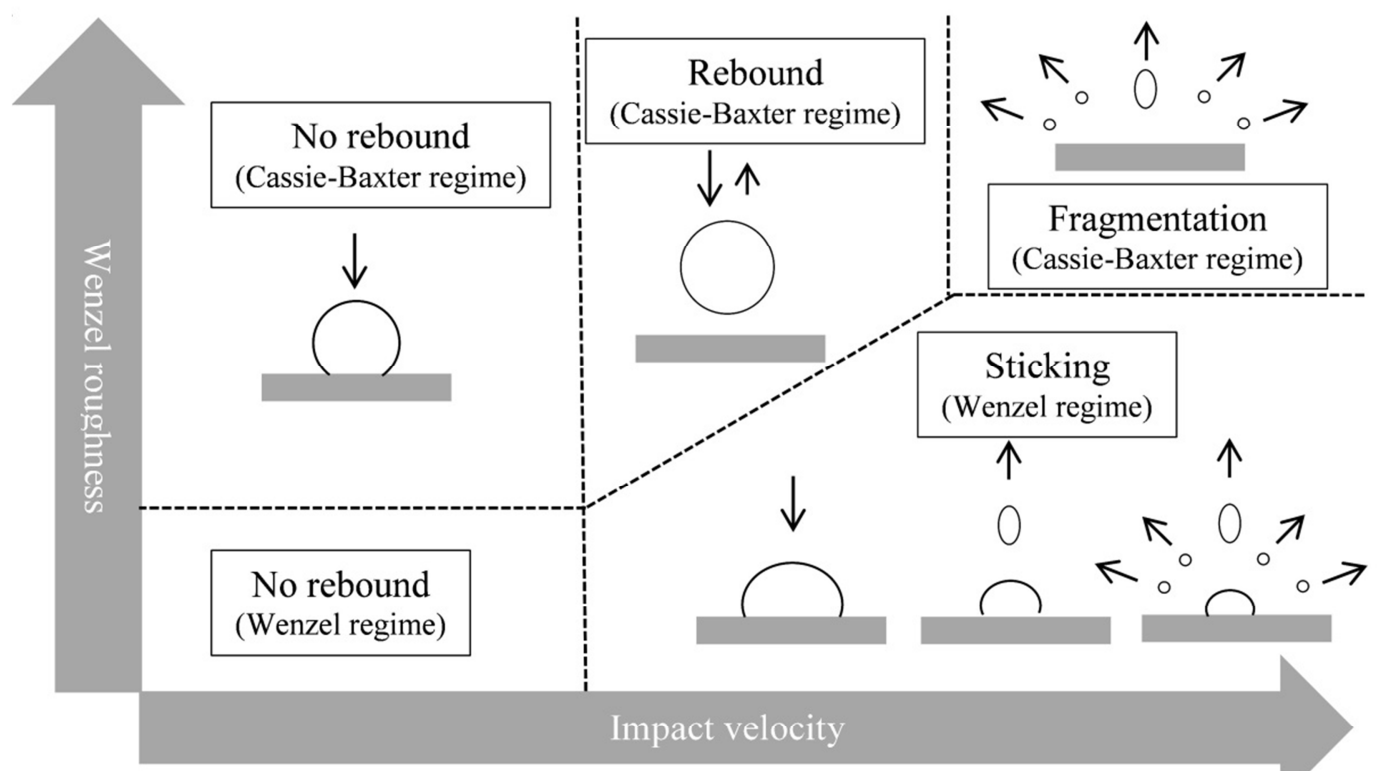

Figure 2

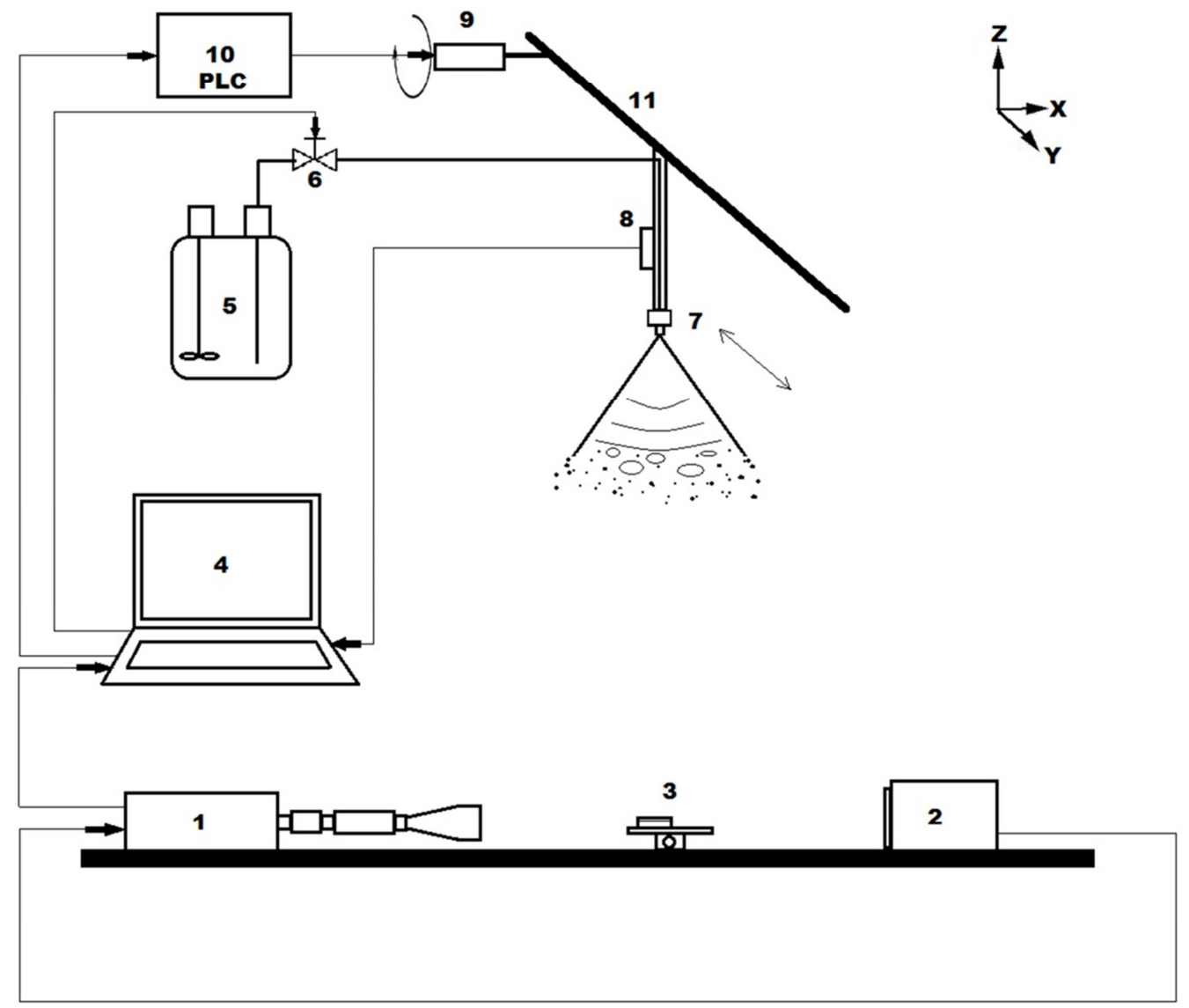


Figure3

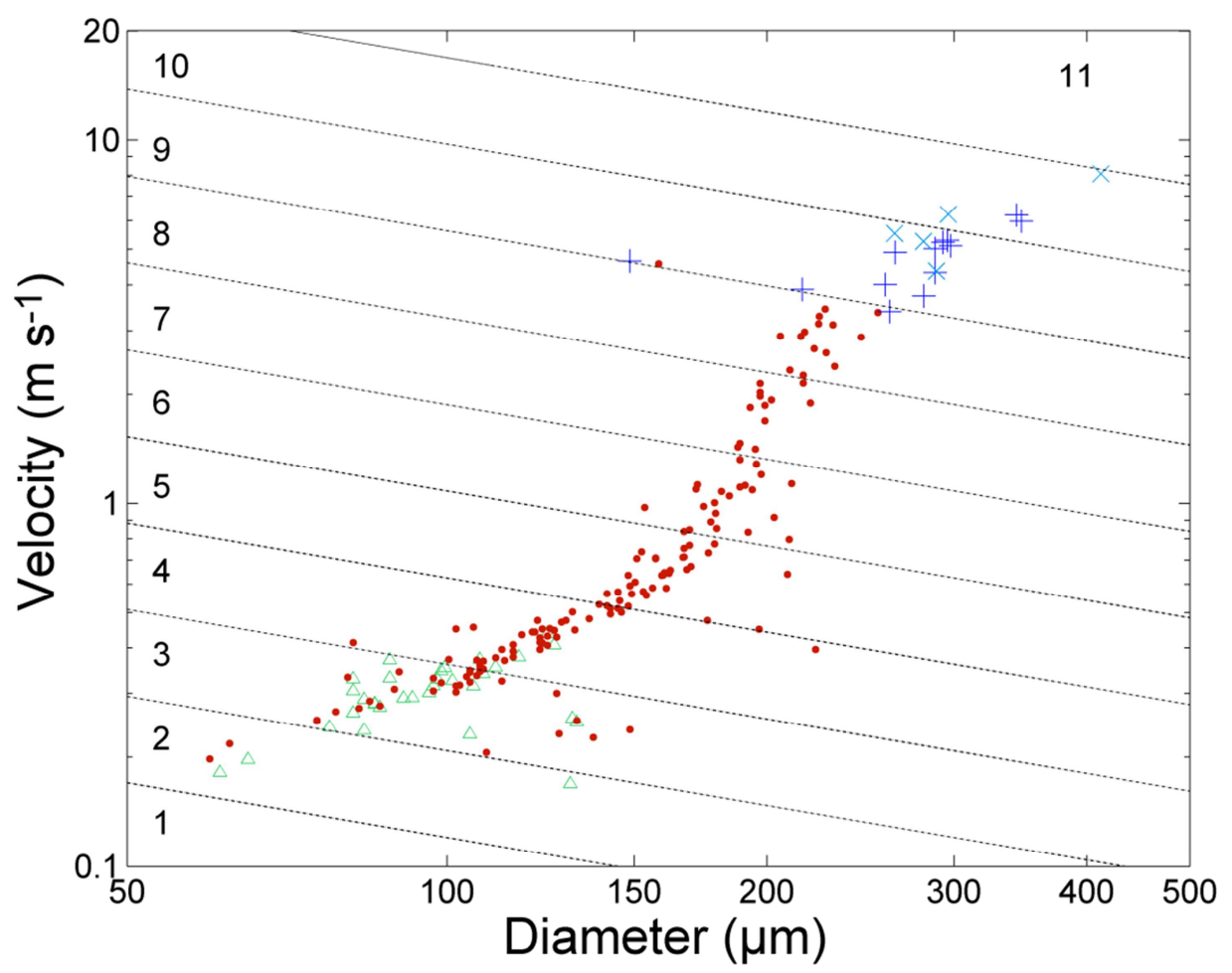

Figure 4

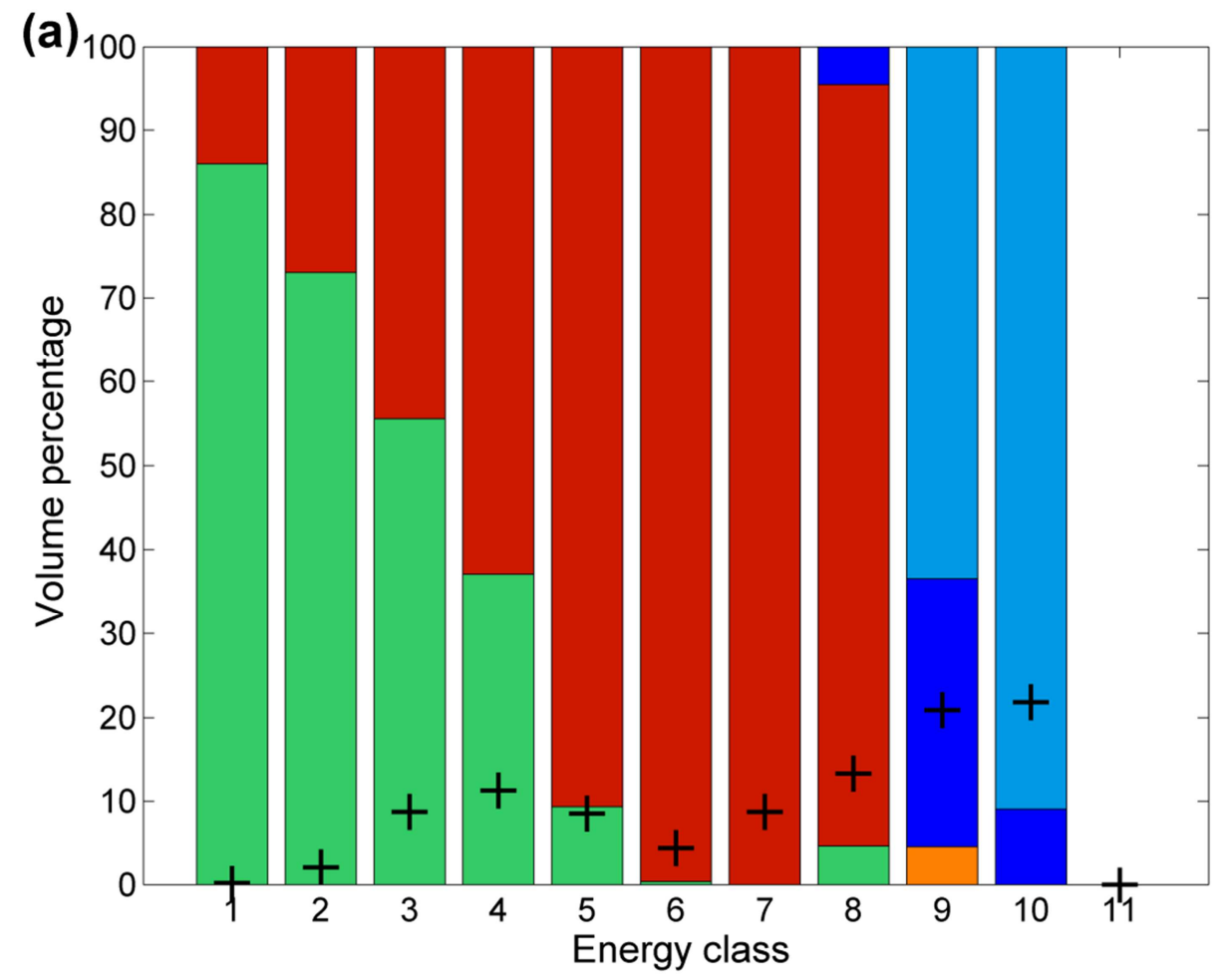



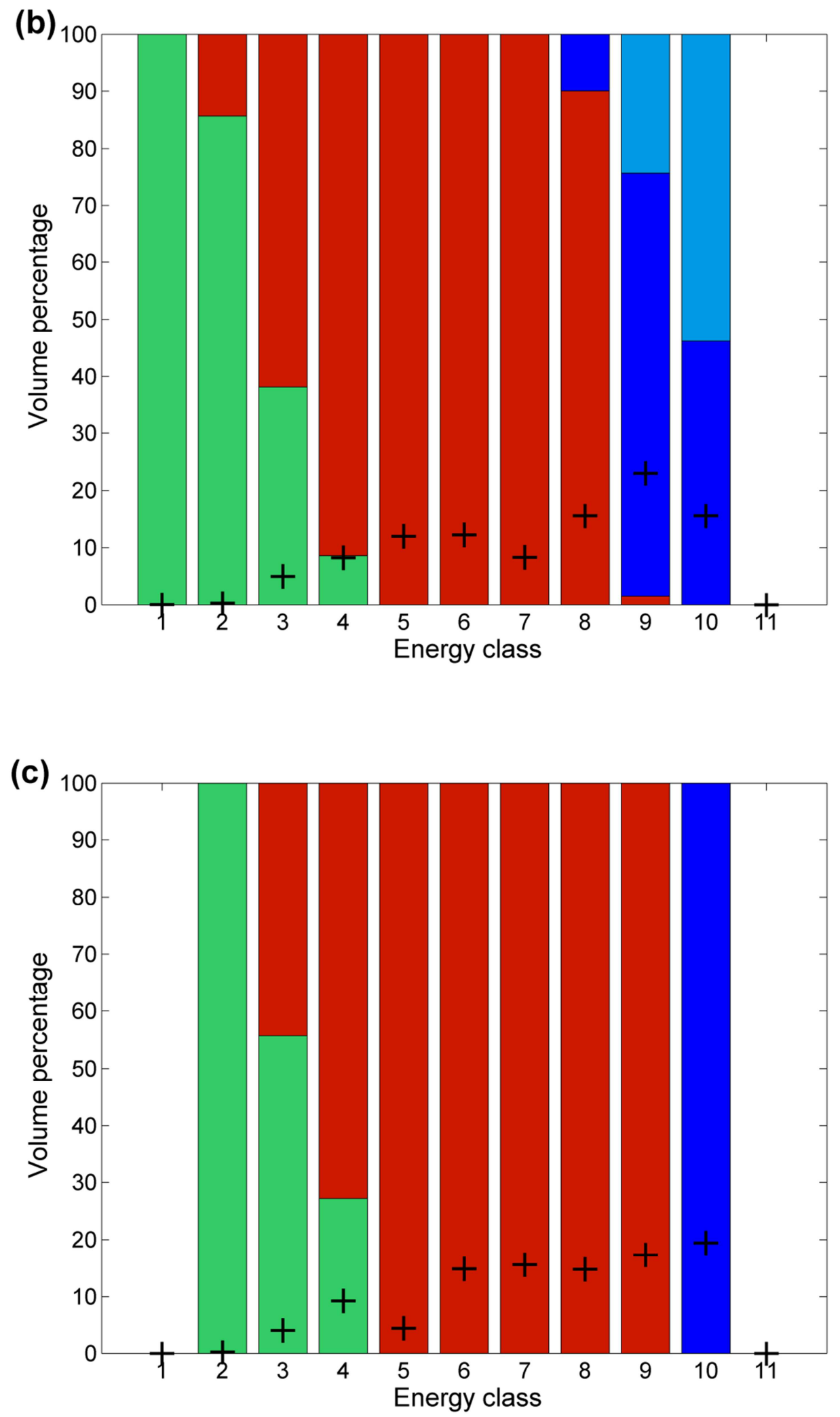


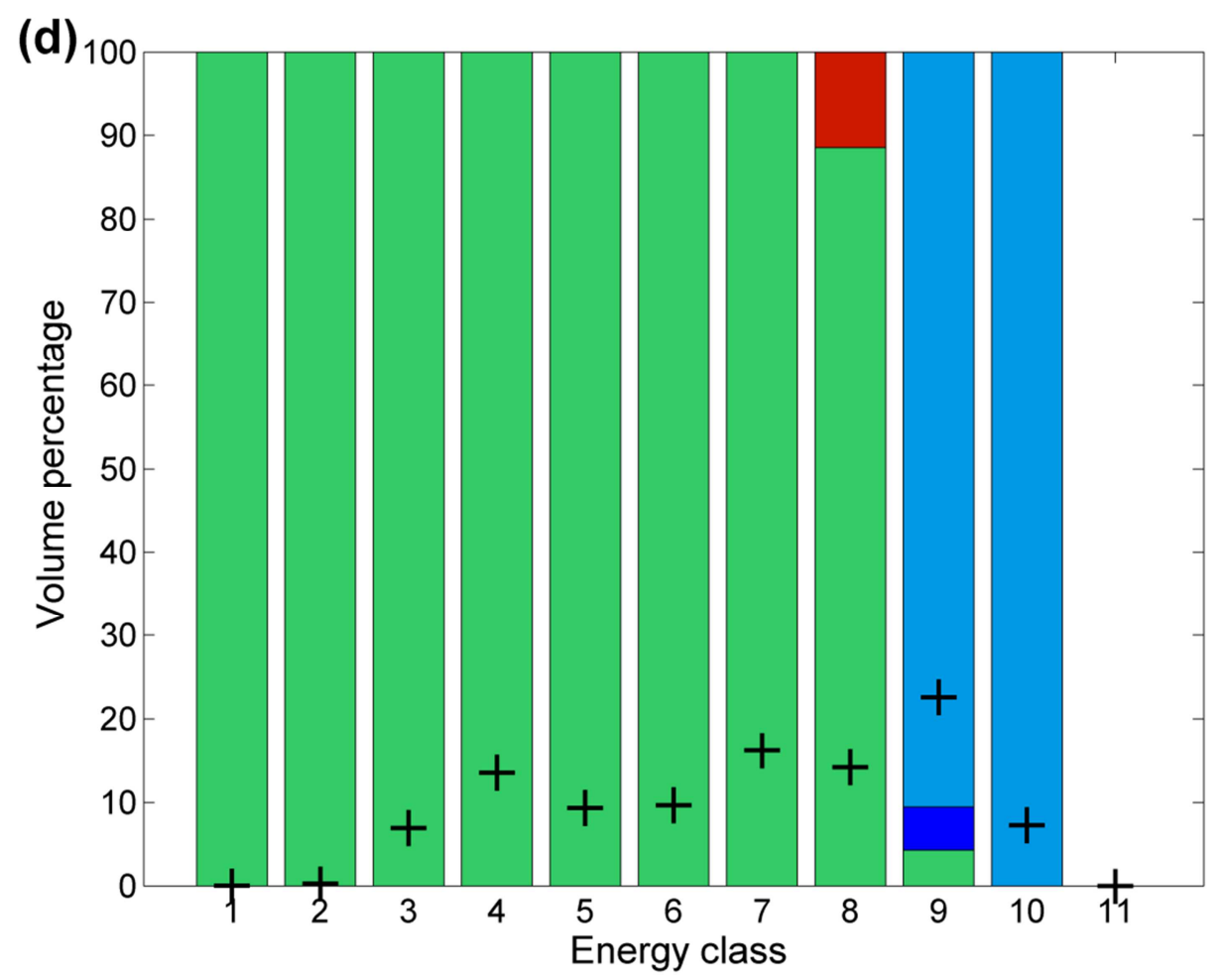



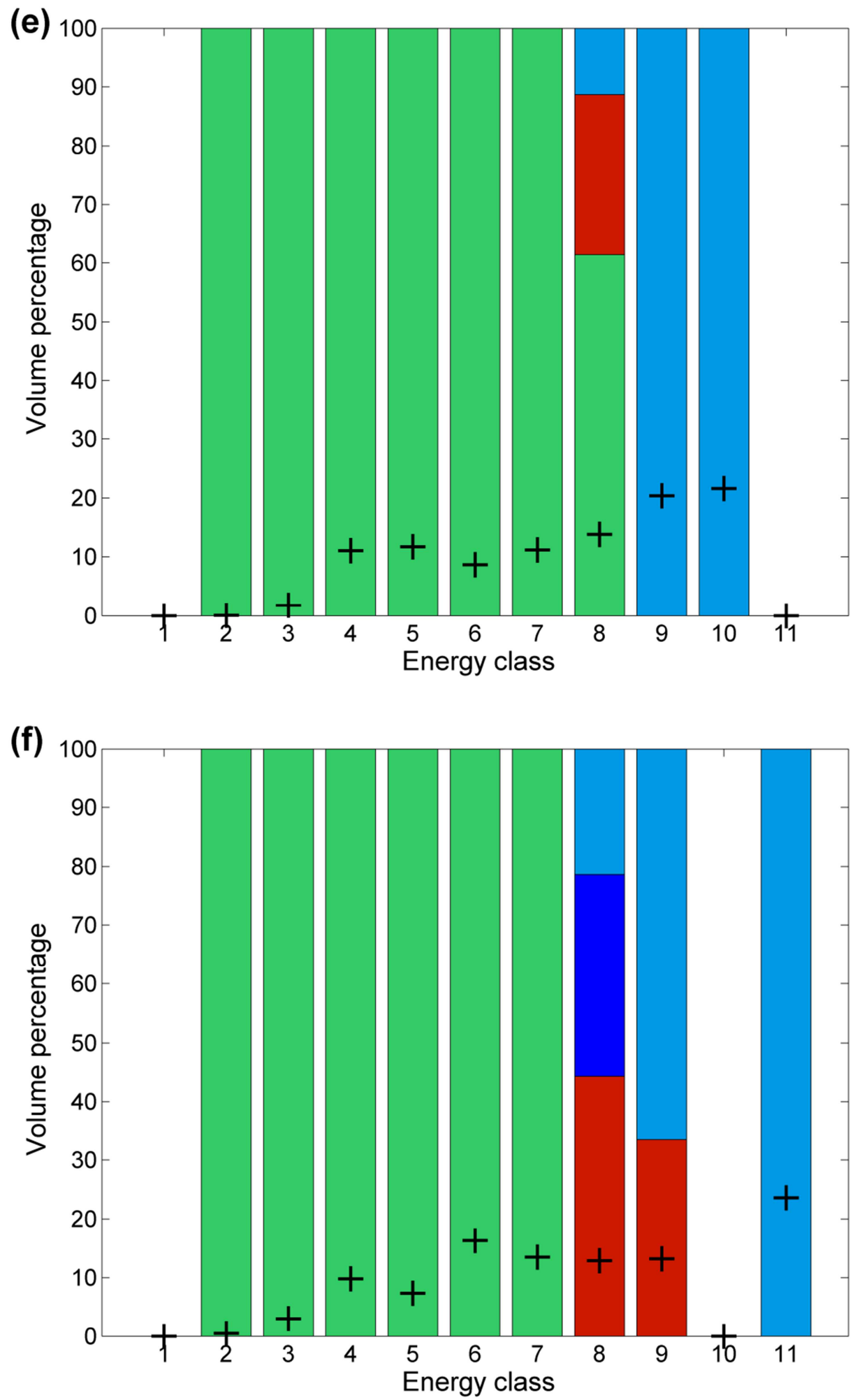